\title{
Simplified Design Methodology for a Slotless Brushless DC Motor
}

\author{
Miroslav Markovic and Yves Perriard \\ Laboratory of Integrated Actuators (LAI), Ecole Polytechnique Fédérale de Lausanne (EPFL), \\ ELG-Ecublens 1015 Lausanne, Switzerland
}

\begin{abstract}
The paper presents a simplified analytical method to design a small two-pole slotless brushless dc motor. Using only a few approximations, the motor analytical model is formulated to generate a system of equations. The system can be solved analytically, producing a motor design for given specifications. The method is used to design a motor with the specifications $150 \mathrm{~W}, 10000 \mathrm{rpm}$, and $18 \mathrm{~V}$.
\end{abstract}

Index Terms-BLDC motor, magnetic field, slotless motor.

\section{INTRODUCTION}

$\mathbf{T}$ HE goal of the paper is to apply an analytical model of a small slotless brushless dc (BLDC) motor, in order to design it according to the given specifications. The motor analytical model is obtained by solving and integrating the permanent-magnet (PM) field.

Normally, it is then possible to embed this model in a mathematical optimization software package and to obtain the design. However, these packages in general can be difficult to use: as it is known to each designer, an analytical model is easy to embed, but there always remains a problem how to choose the initial values and constraints for each free parameter. In order to design a motor, the designer should have a vague idea of the possible values of the motor optimal parameters.

This paper proposes a simple solution to overcome this problem, as it enables designer to rapidly obtain the motor design analytically. By applying an approximation, the motor model is transformed so that it generates a system of equations to solve. For a desired efficiency $\eta$, by varying two free parameters, the system is analytically solved. The solution corresponding to a minimal motor mass $m$ is assumed as the optimum for given $\eta$, and the same procedure is performed for other values of $\eta$. Finally, by choosing a point from the obtained set of nondominant points in the plane $\eta-m$, the first motor design for the given specifications is obtained.

At the second stage, it is possible to "fine-tune" the design, by applying an optimization software and taking the obtained parameters as the initial values. The inclusion of a motor thermal model is a must at this stage.

In the literature, there are some interesting examples of a simplified BLDC motor design. In [1], a design method of applying a parametric analysis is presented. However, the motor mathematical model is not included. The parametric analysis becomes problematic if more than two parameters are to be varied. Reference [2] presents a straightforward design procedure for a slotted motor, but the impression is that the slotted motor model is too approximate: for example, it is not clear how to account for the slotting effect.

Digital Object Identifier 10.1109/TMAG.2006.884108

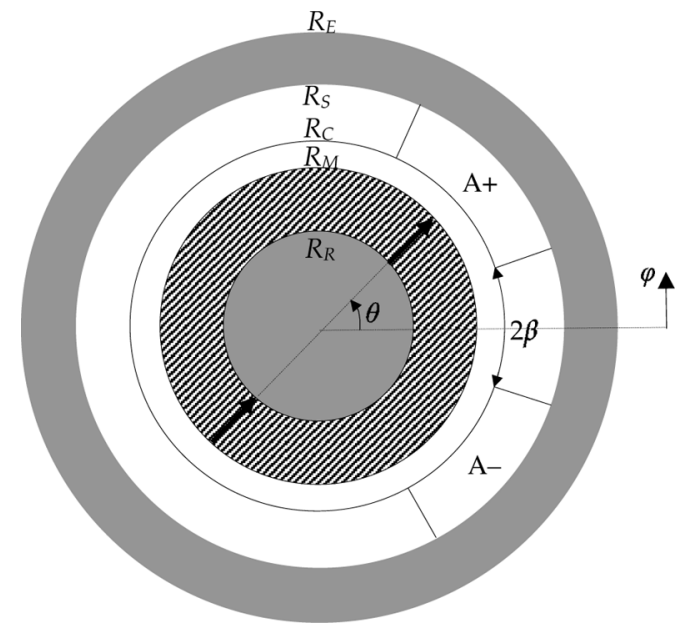

Fig. 1. Cross section of the analyzed two-pole slotless BLDC motor (only the phase $\mathrm{A}$, consisting of the winding sides $\mathrm{A}+$ and $\mathrm{A}-$, is presented).

Recently, many efforts are being made toward coupling optimization algorithms with finite-element method (FEM), but there are not yet reports that it is fully achieved. In [3], the magnet dimensions are chosen analytically using an approximate modeling function, and then the motor is optimized using FEM. In [4], an interesting approach is presented: the FEM is applied to determine how a motor output parameter varies in function of three input parameters (response surface method), and finally a genetic algorithm is used for the optimization in terms of these variables. However, it is not clear how to choose other motor variables, for example the stator slots dimensions.

\section{Motor Configuration to Design}

We designed a two-pole, three-phase slotless motor, with single layer short-pitch windings. The motor is shown in Fig. 1. The rotor angular position is denoted by $\theta$. The field excitation is a parallely magnetized hollow cylinder mounted on a ferromagnetic shaft.

The motor geometric parameters are: rotor yoke (and shaft) radius $R_{R}$, PM radius $R_{M}$, stator internal radius $R_{C}$, stator yoke internal radius $R_{S}$, stator yoke external radius $R_{E}$, and the axial length $l$.

Fig. 1 shows the phase A of the motor. It covers $120^{\circ}$, with the opening angle $2 \beta$ between the positive $(\mathrm{A}+)$ and negative 
(A-) winding sides. Each side (section) contains $N$ conductors carrying the phase current $i$, therefore the number of turns per phase is $N$.

The PM material is described by the remanency $B_{R}$ and the relative recoil permeability $\mu_{r}$. Concerning the stator and rotor yokes' iron materials, the values of the saturation flux density $B_{s, \text { sat }}$ and $B_{r, \text { sat }}$ are known. The mass densities of the PM, iron and copper $\gamma_{\mathrm{pm}}, \gamma_{\mathrm{fe}}$, and $\gamma_{\mathrm{cu}}$ are also known.

The motor specifications are the torque $M$, the speed $\omega=$ $d \theta / d t$, and the amplitude of the phase-to-phase back electromotive force (emf) $E_{l}$ at this speed. The quantity $E_{l}$ has the order of magnitude as the needed dc voltage. In addition, a desired motor efficiency is $\eta$.

The shaft radius $R_{R}$ is considered to be known, as it is determined after a mechanical calculation. The external motor radius $R_{E}$ is also known, as it is determined by the room previewed for the motor. Finally, the mechanical air gap $\delta=R_{C}-R_{M}$ is assumed equal to a technically achievable minimum.

\section{BLDC MOTOR MODEL}

After solving Poisson's equation for the PM magnetic field [5] in the polar coordinate system $(r, \varphi)$, the final solution for the radial air gap field is given by

$$
B_{r}=B_{e}\left(\frac{R_{S}^{2}}{r^{2}}+1\right) \cos (\varphi-\theta)
$$

where

$$
B_{e}=\frac{\left(R_{M}^{2}-R_{R}^{2}\right) R_{M}^{2}}{\left(\mu_{r}+1\right) R_{M}^{2}\left(R_{S}^{2}-R_{R}^{2}\right)+\left(\mu_{r}-1\right)\left(R_{S}^{2} R_{R}^{2}-R_{M}^{4}\right)} B_{R} .
$$

The amplitude of the stator yoke field is

$$
B_{s m}=\frac{2\left(R_{E}^{2}+R_{S}^{2}\right)}{R_{E}^{2}-R_{S}^{2}} B_{e}
$$

The amplitude of the rotor yoke field is

$$
B_{\mathrm{rm}}=\frac{\left(\mu_{r}+1\right) R_{M}^{2}-\left(\mu_{r}-1\right) R_{S}^{2}}{R_{M}^{2}} B_{e}
$$

The torque $m$ corresponding to the phase A, carrying current $i$, is determined by Laplace's force acting on its conductors. Therefore, it is given by

$$
m=l i \frac{N}{S}\left(\int_{\mathrm{A}+} B_{r} r d S-\int_{\mathrm{A}-} B_{r} r d S\right)
$$

where

$$
S=\left(R_{S}^{2}-R_{C}^{2}\right) \frac{\pi / 3-\beta}{2}
$$

is the surface area of one winding side $(\mathrm{A}+$ or $\mathrm{A}-$, as shown in Fig. 1). Similarly, the emf $e$ induced in the phase $\mathrm{A}$ is, using $b l v$ rule, given by

$$
e=l \omega \frac{N}{S}\left(\int_{\mathrm{A}+} B_{r} r d S-\int_{\mathrm{A}-} B_{r} r d S\right)=\omega \frac{m}{i} .
$$

Using the flux $\psi$ induced in the phase $\mathrm{A}$, this emf is given by $e=d \psi / d t=\omega d \psi / d \theta$.

After the integration over the two phase winding sides, the next formulas are obtained

$$
\begin{aligned}
m & =\Psi i \sin \theta \\
e & =\Psi \omega \sin \theta
\end{aligned}
$$

where

$$
\Psi=l N B_{e} R_{e}
$$

is the amplitude of the flux induced in the phase, with

$$
R_{e}=\frac{2}{3} \frac{4 R_{S}^{3}-3 R_{S}^{2} R_{C}-R_{C}^{3}}{R_{S}^{2}-R_{C}^{2}} \frac{2 \cos \beta-1}{\pi / 3-\beta}
$$

and $\psi=-\Psi \cos \theta$

If the three phases are supplied by an appropriate three-phase system of currents with a root-mean-square value $I$, each in phase with the corresponding emf, the total motor torque a constant. Using (8) and superposition, it is given by

$$
M=\frac{3 \sqrt{2}}{2} \Psi I \text {. }
$$

From (9) it follows that the amplitude of the phase-to-phase back emf is

$$
E_{l}=\sqrt{3} \Psi \omega
$$

This model is valid only in the case, if the stator and rotor iron can be considered as ideal (with infinite permeability). To satisfy this condition, experience shows than the iron flux density amplitude should not surpass $90 \%$ of its saturation level. This assumption will be verified later. It gives two conditions: $B_{s m}<0.9 B_{s, \text { sat }}$ and $B_{\text {rm }}<0.9 B_{r, \text { sat }}$.

The conductor used to wind phase $\mathrm{A}$ has the length

$$
l_{c}=N\left[2 l+\left(R_{S}+R_{C}\right)(\pi / 3+\beta)\right]
$$

and the cross section area $S_{c}=k_{f} S / N$, with $S$ given by (6) and $k_{f}$ the filling factor (considered to be known). It gives

$$
S_{c}=\frac{k_{f}\left(R_{S}^{2}-R_{C}^{2}\right)(\pi / 3-\beta)}{2 N} .
$$

Therefore, the phase resistance is given by

$$
R=\varrho \frac{l_{c}}{S_{c}}=\frac{2 \varrho N^{2}\left[2 l+\left(R_{S}+R_{C}\right)(\pi / 3+\beta)\right]}{k_{f}\left(R_{S}^{2}-R_{C}^{2}\right)(\pi / 3-\beta)}
$$

where $\varrho$ is the copper specific resistance.

The equation of the power balance is given by

$$
P_{\mathrm{cu}}+P_{\mathrm{fe}}=\frac{1-\eta}{\eta} M \omega
$$

where

$$
P_{\mathrm{cu}}=3 R I^{2}
$$

are the copper losses, and

$$
P_{\mathrm{fe}}=p_{\mathrm{fe}} m_{\mathrm{fe}, s}=p_{\mathrm{fe}} \gamma_{\mathrm{fe}}\left(R_{E}^{2}-R_{S}^{2}\right) \pi l
$$


are the stator iron losses, where

$$
p_{\mathrm{fe}}=C_{f}\left(\frac{f}{50}\right)^{k} B_{s m}^{2}=C_{\omega} \omega^{k} B_{s m}^{2}
$$

are the specific losses [6] in $\mathrm{W} / \mathrm{kg}$, and $m_{\mathrm{fe}, s}$ is the stator iron mass. The parameters $C_{f}$ and $k$ are obtained from the manufacturer's catalog, and $C_{\omega}$ is calculated. The other motor losses (e.g., friction) are neglected.

The active motor mass

$$
m=m_{\mathrm{fe}}+m_{\mathrm{pm}}+m_{\mathrm{cu}}
$$

is a sum of the iron mass

$$
m_{\mathrm{fe}}=m_{\mathrm{fe}, r}+m_{\mathrm{fe}, s}=\gamma_{\mathrm{fe}}\left[R_{R}^{2}+\left(R_{E}^{2}-R_{S}^{2}\right)\right] \pi l
$$

PM mass

$$
m_{\mathrm{pm}}=\gamma_{\mathrm{pm}}\left(R_{M}^{2}-R_{R}^{2}\right) \pi l
$$

and copper mass

$$
m_{\mathrm{cu}}=3 \gamma_{\mathrm{cu}} S_{c} l_{c}=3 \gamma_{\mathrm{cu}} N S_{c}\left[2 l+\left(R_{S}+R_{C}\right)(\pi / 3+\beta)\right] .
$$

Finally, the relation between the parameters $R_{C}$ and $R_{M}$

$$
R_{C}=R_{M}+\delta
$$

completes the motor mathematical model.

\section{SySTEM OF EQUATIONS}

Applying a formal approach, the parameters $M, \omega, E_{l}, \eta, k_{f}$, $R_{R}, R_{E}, \delta, B_{R}, \mu_{r}, \gamma_{\mathrm{pm}}, B_{s, \mathrm{sat}}, k, C_{\omega}, B_{r, \mathrm{sat}}, \gamma_{\mathrm{fe}}, \varrho$, and $\gamma_{\mathrm{cu}}$ from the model in Section III are known. It will be shown how to transform the model to generate a cubic equation with two free parameters.

Knowing $E_{l}$ and $\omega, \Psi$ is determined from (13), and $I$ is determined from (12). From now on, the parameters $\beta$ and $B_{\text {rm }}$ will be treated as free parameters, which will produce a parametric analysis with two parameters to vary. These two parameters are chosen only due to the fact that it enables an analytical solution of the system of equations.

Then, in order to maximally use the stator iron material, the field amplitude in the stator should be equal to $90 \%$ of its saturation level

$$
B_{s m}=0.9 B_{s, \text { sat }}
$$

The condition $B_{\mathrm{rm}}<0.9 B_{r \text {,sat }}$ remains valid, and it should be always satisfied (it can not become an equation, as $R_{R}$ is determined after a mechanical calculation). Due to this fact, when performing the parametric analysis, this condition will determine the maximal value for the parameter $B_{\mathrm{rm}}$.

By assuming approximately that for the PM material $\mu_{r}=1$ (which will be justified later), from (4) it follows

$$
B_{e}=\frac{B_{\mathrm{rm}}}{2} \text {. }
$$

TABLE I

MOTOR AND MATERIALS SPECIFICATIONS

\begin{tabular}{|c|l|l|}
\hline$M$ & $143 \mathrm{mNm}$ & nominal motor torque \\
\hline$\omega$ & $10000 \mathrm{rpm}$ & nominal motor speed \\
\hline$E_{l}$ & $18 \mathrm{~V}$ & nominal motor back emf \\
\hline$\eta$ & variable & motor efficiency \\
\hline \hline$k_{f}$ & 0.4 & filling factor \\
\hline$R_{R}$ & $4 \mathrm{~mm}$ & shaft radius \\
\hline$R_{E}$ & $25 \mathrm{~mm}$ & motor external radius \\
\hline$\delta$ & $0.5 \mathrm{~mm}$ & mechanical air gap \\
\hline$B_{R}$ & $1.28 \mathrm{~T}$ & PM remanency \\
\hline$\mu_{r}$ & 1.03 & PM relative permeability \\
\hline$\gamma_{p m}$ & $7700 \mathrm{~kg} / \mathrm{m}^{3}$ & PM density \\
\hline$B_{s, s a t}$ & $1.52 \mathrm{~T}$ & stator iron saturation flux density \\
\hline$k$ & 1.406 & stator iron losses coefficient \\
\hline$C_{f}$ & $0.9677 \mathrm{~W} / \mathrm{kg} / \mathrm{T}^{2}$ & stator iron losses coefficient \\
\hline$B_{r, s a t}$ & $0.85 \mathrm{~T}$ & rotor iron saturation flux density \\
\hline$\gamma_{f e}$ & $7650 \mathrm{~kg} / \mathrm{m}^{3}$ & stator and rotor iron density \\
\hline$\varrho$ & $1.72 \cdot 10^{-8} \Omega \mathrm{m}$ & copper specific resistivity \\
\hline$\gamma_{c u}$ & $8900 \mathrm{~kg} / \mathrm{m}^{3}$ & copper density \\
\hline
\end{tabular}

Substituting this $B_{e}$ in (3) and (2) (again with $\mu_{r}=1$ ), it follows

$$
\begin{aligned}
R_{S} & =R_{E} \sqrt{\frac{B_{s m}-B_{\mathrm{rm}}}{B_{s m}+B_{\mathrm{rm}}}} \\
R_{M} & =\sqrt{R_{R}^{2}+\left(R_{S}^{2}-R_{R}^{2}\right) \frac{B_{\mathrm{rm}}}{B_{R}}} .
\end{aligned}
$$

Finally, $R_{C}, R_{e}$, and $p_{\mathrm{fe}}$ are determined using (25), (11), and (20), respectively.

In order to determine the remaining unknowns $l$ and $N$, we proceed as follows. From (10), it follows

$$
N=\frac{\Psi}{l B_{e} R_{e}} .
$$

Substituting $N$ in (16), and then $R$ in the power balance (17), it follows

$$
\begin{aligned}
& 3 I^{2} \frac{2 \varrho\left[2 l+\left(R_{S}+R_{C}\right)(\pi / 3+\beta)\right]}{k_{f}\left(R_{S}^{2}-R_{C}^{2}\right)(\pi / 3-\beta)} \frac{\Psi^{2}}{l^{2} B_{e}^{2} R_{e}^{2}} \\
& +p_{\mathrm{fe}} \gamma_{\mathrm{fe}}\left(R_{E}^{2}-R_{S}^{2}\right) \pi l=\frac{1-\eta}{\eta} M \omega .
\end{aligned}
$$

The last equation gives a cubic equation for $l$

$$
A l^{3}-B l^{2}+C l+D=0
$$

with the coefficients

$$
\begin{aligned}
A= & p_{\mathrm{fe}} \gamma_{\mathrm{fe}}\left(R_{E}^{2}-R_{S}^{2}\right) \pi \\
B= & \frac{1-\eta}{\eta} M \omega \\
C= & \frac{12 \varrho I^{2} \Psi^{2}}{k_{f}\left(R_{S}^{2}-R_{C}^{2}\right)(\pi / 3-\beta) B_{e}^{2} R_{e}^{2}} \\
D= & \frac{6 \varrho I^{2} \Psi^{2}}{k_{f}\left(R_{S}^{2}-R_{C}^{2}\right)(\pi / 3-\beta) B_{e}^{2} R_{e}^{2}} \\
& \times\left(R_{S}+R_{C}\right)(\pi / 3+\beta) .
\end{aligned}
$$




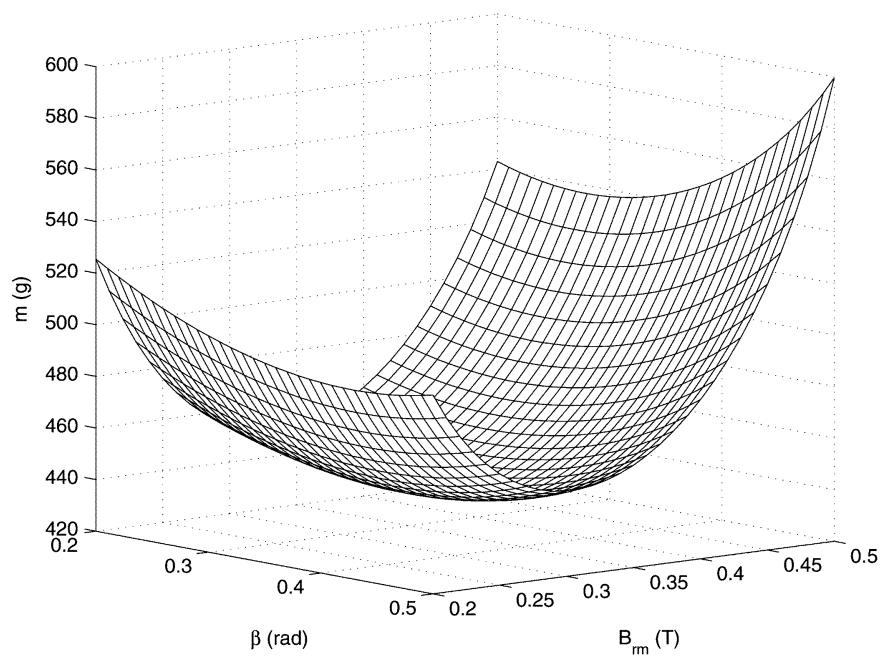

Fig. 2. The motor mass $m$ versus the parameters $\beta$ and $B_{\mathrm{rm}}$ for $\eta=94 \%$.

TABLE II

MINIMA OF THE FUNCTION $m=m\left(\beta, B_{\mathrm{rm}}\right)$ FOR VARIOUS $\eta$

\begin{tabular}{||c||c|c|c|c|c|c|}
\hline$\eta(\%)$ & 90 & 91 & 92 & 93 & 94 & 95 \\
\hline$\beta(\mathrm{rad})$ & 0.36 & 0.35 & 0.35 & 0.35 & 0.35 & 0.33 \\
\hline$B_{r m}(\mathrm{~T})$ & 0.36 & 0.36 & 0.35 & 0.34 & 0.32 & 0.29 \\
\hline$m(\mathrm{~g})$ & 278 & 301 & 332 & 374 & 439 & 573 \\
\hline
\end{tabular}

Its solution is $l$, and finally $N$ is determined using (30). From three solutions of (32), we choose the minimal real one. Therefore, the motor design is completed, and all the motor parameters are determined.

\section{DEsign EXAMPLE}

The motor specifications are given in Table I, along with the chosen materials' properties. The motor is intended to drive an artificial respirator.

The procedure is as follows: for an efficiency $\eta$, the methodology from Section IV is applied by varying $\beta$ and $B_{\mathrm{rm}}$ in two nested loops, with necessary limits and steps, which produces a set of solutions. The result corresponding to $\eta=94 \%$ is presented in Fig. 2. Finally, the solution corresponding to a minimal mass is accepted. This simulation is very fast (it lasts a couple of seconds).

For another efficiency, another set of solutions is obtained, and the minimum is achieved for new values of $\beta$ and $B_{\mathrm{rm}}$. The corresponding minima are presented in Table II and Fig. 3.

Finally, the obtained curve $m(\eta)$ in Fig. 3, represents a set of nondominated points: each of them can be treated as a solution. In the multiobjective optimization theory, such a curve is called trade-off (or Pareto) curve. Which solution to assume is now question of other factors. Due to some economical reasons, the solution for $\eta=94 \%$ is chosen as the final one. The corresponding parameters are summarized in Table III.

\section{VERIFICATIONS}

The calculus using the equations from Section III with the real PM permeability $\mu_{r}=1.03$ gives the efficiency $\eta=93.9 \%$, therefore the only approximation $\mu_{r} \simeq 1$ is justified.

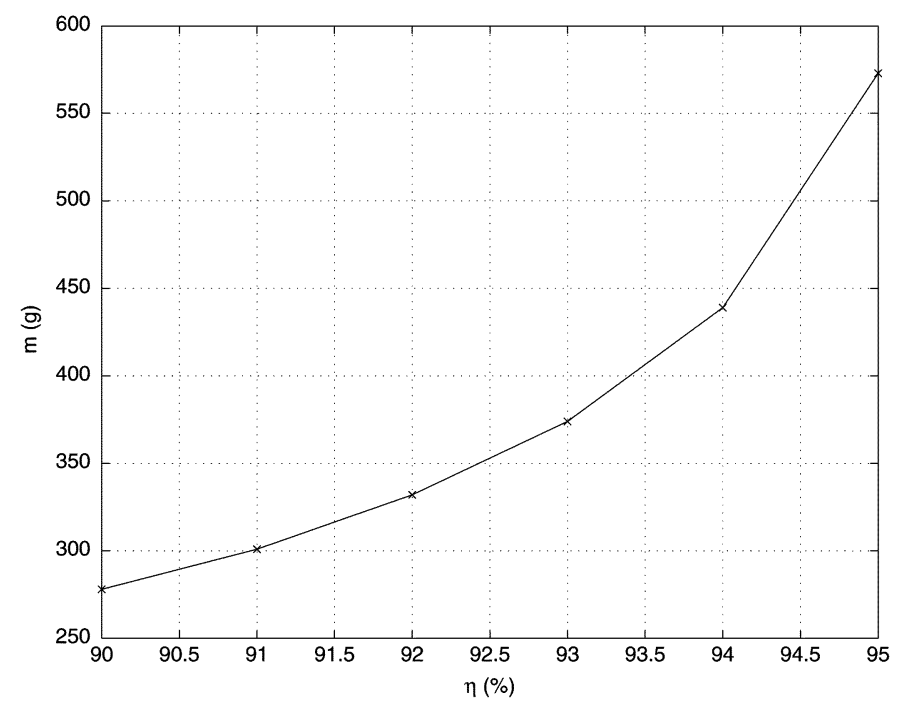

Fig. 3. The minima of the motor mass $m$ versus the motor efficiency $\eta$.

TABLE III

MOTOR FINAL DESIGN

\begin{tabular}{|c|l|l|}
\hline$R_{R}$ & $4.0 \mathrm{~mm}$ & shaft radius \\
\hline$R_{M}$ & $10.4 \mathrm{~mm}$ & PM radius \\
\hline$R_{C}$ & $10.9 \mathrm{~mm}$ & stator internal radius \\
\hline$R_{S}$ & $19.7 \mathrm{~mm}$ & stator yoke internal radius \\
\hline$R_{E}$ & $25.0 \mathrm{~mm}$ & stator yoke external radius \\
\hline$l$ & $38.4 \mathrm{~mm}$ & axial lenght \\
\hline$N$ & 31 & number of turns/phase \\
\hline$S_{c}$ & $1.2 \mathrm{~mm}^{2}$ & conductor cross section area \\
\hline$m$ & $439 \mathrm{~g}$ & motor active mass \\
\hline
\end{tabular}

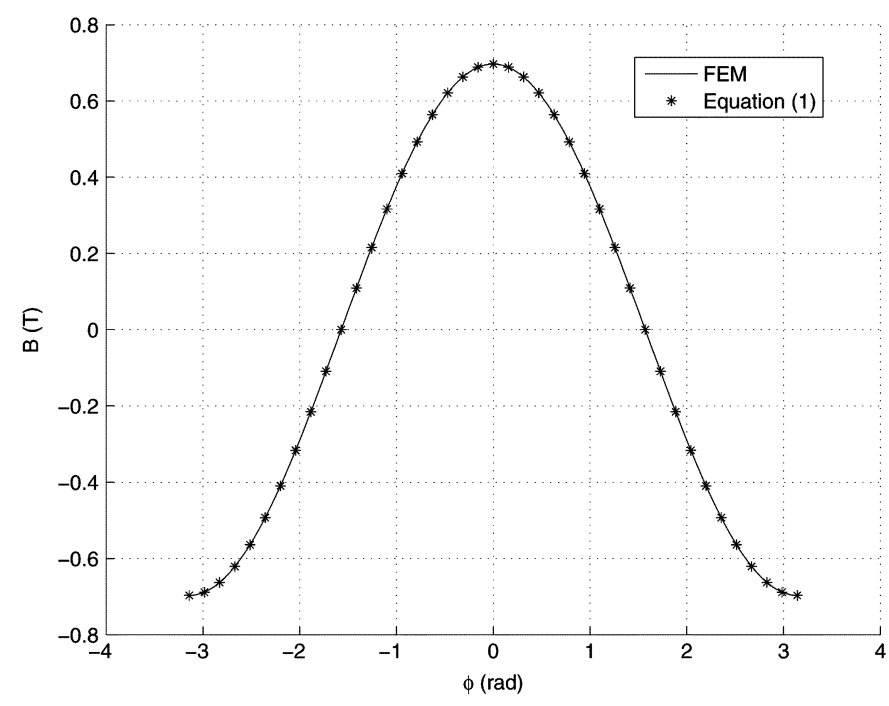

Fig. 4. The air gap field along the line $r=10.6 \mathrm{~mm},-\pi<\varphi<\pi$.

The analysis using a FEM commercial software gives the value $9.643 \mathrm{mWb}$ of the phase flux amplitude $\Psi$, compared to $9.647 \mathrm{mWb}$ calculated using (10) (which gives the error of $0.04 \%)$.

The solution for the air gap field obtained using (1) matches FEM result as well: Fig. 4 shows the air gap field along the circular line with $r=10.6 \mathrm{~mm}$, for $-\pi<\varphi<\pi$. It justifies the approximation that the iron can be treated as ideal, if its flux 


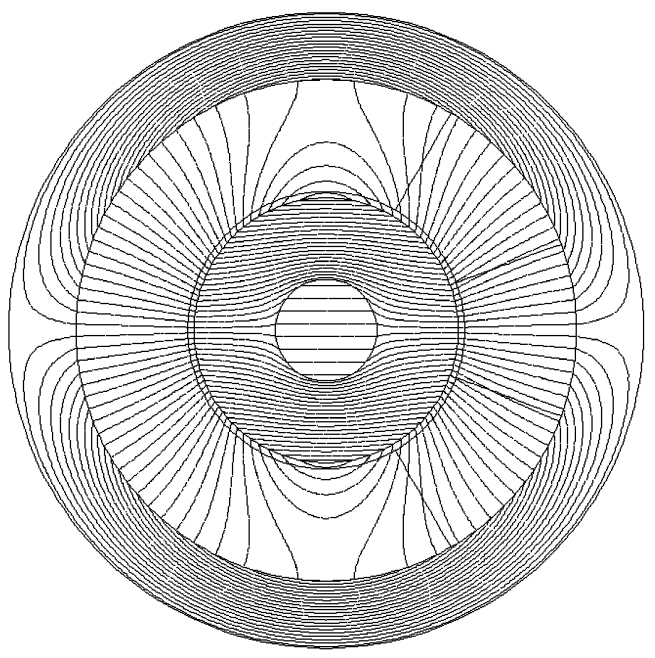

Fig. 5. The motor cross section with the PM flux lines for $\theta=0$.

density amplitude does not surpass $90 \%$ of its saturation level. Fig. 5 shows the PM flux lines obtained using the FEM software.

\section{CONCLUSION}

The paper presented a simplified method to design a BLDC motor. Introducing only one approximation in the motor ana- lytical model, a system of equations is created and analytically solved.

The following step in the design would be to apply an optimization software package. The same analytical model (with the thermal motor model included) without any approximation should be used, taking the obtained motor parameters as the initial values.

\section{REFERENCES}

[1] M. Jufer, C. Peclat, and A. Birkicht, "Electromagnetic high speed micromotor," in Proc. 26th Annu. Symp. Incremental Motion Control Systems and Devices (IMCSD), San Jose, CA, Jul. 1997, pp. 159-164.

[2] W. Arshad, Y. Chin, T. Backstrom, J. Soulard, S. Ostlund, and C. Sadarangani, "On finding compact motor solutions for transient applications," in IEEE Electric Machines and Drives Conf., IEMDC, 2001, pp. 743-747.

[3] C. Mi, "Analytic design of permanent magnet traction drive motors," IEEE Trans. Magn., vol. 42, no. 7, pp. 1861-1866, Jul. 2006.

[4] L. Jolly, M. Jabbar, and L. Qinghua, "Design optimization of permanent magnet motors using response surface methodology and genetic algorithms," IEEE Trans. Magn., vol. 41, no. 10, pp. 3928-3930, Oct. 2005.

[5] M. Markovic and Y. Perriard, "Analytical formula for the torque/emf constant of a slotless BLDC motor," presented at the IEEE Conf. Electromagnetic Field Computation, CEFC, 2006, paper 10059.

[6] M. Jufer, Electromecanique. Lausanne, Switzerland: Presses polytechniques et universitaires romandes, 1998.

Manuscript received May 19, 2006; revised September 5, 2006. Corresponding author: M. Markovic (e-mail: miroslav.markovic@epfl.ch). 